\title{
MTT Detection of the Toxicity on CdTe Quantum Dots
}

\author{
Xiong Su ${ }^{1^{*}}$, Xiaoyu Gao ${ }^{2 *}$, Li Jiang ${ }^{*}$, Jing Zhang ${ }^{1}$, Zhifang Wang1, Baofeng Chi', Hairong Zhang1, \\ Shibo Bao1, Ran Zhang1, Baihui Yun', Dejun Sun"\#, Juan Sun"\# \\ ${ }^{1}$ Inner Mongolia Medical University, Hohhot, China \\ ${ }^{2}$ Inner Mongolia People's Hospital, Hohhot, China \\ ${ }^{3}$ South China Institute of Software Engineering, Guangzhou, China \\ ${ }^{4}$ Inner Mongolia Honder College of Arts and Sciences, Hohhot, China \\ Email: \#dejunsun123456@163.com, "sj6840@163.com
}

How to cite this paper: Su, X., Gao, X.Y., Jiang, L., Zhang, J., Wang, Z.F., Chi, B.F., Zhang, H.R., Bao, S.B., Zhang, R., Yun, B.H., Sun, D.J. and Sun, J. (2021) MTT Detection of the Toxicity on CdTe Quantum Dots. Open Journal of Preventive Medicine, 11, 1-8.

https://doi.org/10.4236/ojpm.2021.111001

Received: November 6, 2020

Accepted: January 2, 2021

Published: January 5, 2021

Copyright $\odot 2021$ by author(s) and Scientific Research Publishing Inc. This work is licensed under the Creative Commons Attribution International License (CC BY 4.0).

http://creativecommons.org/licenses/by/4.0/

\begin{abstract}
Objective: The quantum dots are the useful materials in microelectronics and biomedical research. However its toxicity has to be considered. We studied the effect of cell inhibition with ZnS core quantum dots and CdTe quantum dots modified with Thioglycolic acid or Cysteine functional group (TGA-CdTe, TGA-CdTe/ZnS, Cys-CdTe, Cys-CdTe/ZnS) on Caco-2 cell proliferation. Methods: We studied the effect of cell inhibition with ZnS core QDs and CdTe QDs modified with functional group on Caco-2 cell proliferation by MTT assay at $0,12.5,25,50,100 \mu \mathrm{g} / \mathrm{ml}$ and $6,24,48 \mathrm{~h}$. Result: Our results showed that all QDs have inhibited cell proliferation and reached maximum 79.21\%. The inhibition rate of Cys-modified QDs increased with the increase of concentration and reached maximum $66.72 \%$. The inhibition rate of TGA-modified QDs increased with the increase of time. The ratios of Cys-modified to TGA-modified were less than 1 at all concentrations and three exposure times $(\mathrm{P} \leq 0.01)$. The average ratios of Cys-CdTe/ZnS to Cys-CdTe reached 1.11 only for $48 \mathrm{~h}(\mathrm{P} \leq 0.05)$. The ratios of TGA-CdTe/ZnS to TGA-CdTe were closed to 1 at all concentrations and exposure times. Conclusion: The regularity of QDs modified with functional group is that inhibition of TGA-modified higher than Cys-modified. Inhibition exhibited dose-dependent for Cys-modified while exhibited time-dependent for TGA-modified. The regularity of CdTe-QDs with ZnS or not is that the inhibition of Cys-CdTe/ZnS was higher than Cys-CdTe while TGA-CdTe/ZnS and TGA-CdTe were consistent.
\end{abstract}

\section{Keywords}

CdTe Quantum Dots, MTT, Inhibition Rate

*Xiong Su, Xiaoyu Gao, Li Jiang contributed equally to this paper. 


\section{Introduction}

QDs possess unique optical and electronic properties including tunable emission wavelength, broadband absorption spectrum, and photostability that make them useful materials in microelectronics and biomedical research [1]. QDs toxicity and surface coatings to render them biologically compatible have been intensively researched (Rizvi et al., 2012). QDs were appealing alternatives to conventional fluorophores due to their superior optical properties and have the potential to meet some of these outstanding challenges in biotechnology [2].

Cadmium QDs (Cd-QDs) including CdTe-QDs and CdSe-QDs have shown great potential for use as fluorescent tags in therapeutic targeting and in medical and molecular imaging but its toxicity has to be considered [1]. QDs toxicity limits their biomedical applicatio, although they can now be aqueously synthesized. QDs toxicity was multifactorial and was determined by their physiochemical properties, including composition of the core, size, surface charge, concentration, surface chemistry, bioactivity, oxidative, photolytic and mechanical stability, as well as their environmental interactions [3] [4]. Cd-QDs toxicity has been intensively researched. The toxicity of Cd-based QDs has been proposed to be associated with the oxidation reaction of the metal core. The reaction generates reactive oxygen species (ROS) and $\mathrm{Cd}^{2+}$, which were toxic to cells of animals, plants and microbes [5]. Another research found that the physicochemical characteristics of CdTe core QDs influenced subcellular localization and cytotoxicity; quantified as generation of ROS [6]. Cadmium (Cd), which was capable of inducing known toxicities in humans including hepatic, renal, neurologic, and/or genetic toxicities, was the most abundant component of QDs [7]. A research found that CdTe-QDs with a smaller size showed greater hematopoiesis toxicity and CdTe-QDs effects on immune system [5] [8]. CdTe-QDs can induce cytotoxicity, autophagy, oxidative stress, ER stress, chromatin condensation, reducing cell viability and apoptosis in cells [9]. Based on the above research, we intend to study the effect of cell inhibition with CdTe core QDs and CdTe QDs modified with functional group in vitro experiments. Our results provide new insights into Cd-QDs toxicology and provide evidence for the future application.

\section{Materials and Methods}

\section{Preparation and Characteristics of $Q D s$}

The CdTe-QDs used in the study were synthesized by Janus New-Materials Co., Ltd and kept in a refrigerator at $4^{\circ} \mathrm{C}$ until use. The physicochemical properties of these QDs were evaluated. Ultraviolet-visible (UV-vis) spectra were measuring with a PerkinElmer UV-VisNIR (Lambda 900) spectrophotometer (PerkinElmer, Waltham, MA, USA). Photoluminescence spectra were collected with a Cary Eclipse fluorescence spectrometer (Varian Medical Systems, Palo Alto, CA, USA). The concentration of Cysteine (Cys) and Thioglycolic acid (TGA)-modified $\mathrm{CdTe} / \mathrm{ZnS}$ and CdTe QDs aqueous solution was calculated to be $8 \mu \mathrm{mol} / \mathrm{L}$ (3 $\mathrm{mg} / \mathrm{ml}$ ) by UV absorption and Lambert's law. The Emission Voltage was $500 \mathrm{~V}$. 
The emission wavelength of the resulting solution was $450 \mathrm{~nm}$. Emission wavelength was $537 \pm 5 \mathrm{~nm}$. Maximum absorption peaks was $500 \mathrm{~nm}$.

\section{Cell Culture}

Caco- 2 cells were obtained from Fu Heng biology (Shanghai, China) and were maintained in DMEM (Gibco, NY, USA)supplemented with $10 \%$ fetl bovine serum (Gibco, NY, USA) and 1\% penicillin-streptomycin-neomycin (Gibco, NY, USA). Cells were incubated in a humid atmosphere at $37^{\circ} \mathrm{C}$ and $5 \% \mathrm{CO}_{2}$, and passaged with $0.25 \%$ trypsin-EDTA, confluence $70 \%-80 \%$ began subculturing.

Cell Treatment

Confluence of about $80 \%$ of the logarithmic cells was collected. Cells were seeded in 96-well plates at 106 cells/well in $100 \mu \mathrm{l}$ of medium and incubated at 5\% $\mathrm{CO}_{2}$ and $37^{\circ} \mathrm{C}$ for $12 \mathrm{~h}$. Cells were treated to concentration $(0,12.5,25,50,100$ $\mu \mathrm{g} / \mathrm{ml}$ ) of Cys-modified CdTe QDs (Cys-CdTe QDs), Cys-modified CdTe/ZnS QDs (Cys-CdTe/ZnS QDs), TGA-modified CdTe QDs (TGA-CdTe QDs) and TGA-modified CdTe/ZnS QDs (TGA-CdTe/ZnS QDs) at $5 \% \mathrm{CO}_{2}$ and $37^{\circ} \mathrm{C}$ for $6 \mathrm{~h}, 24 \mathrm{~h}$ and $48 \mathrm{~h}$ in the following experiments.

\section{$M T T$}

Cells were incubated with four QDs at $5 \% \mathrm{CO}_{2}$ and $37^{\circ} \mathrm{C}$ for 6,24 , and $48 \mathrm{~h}$. $20 \mu \mathrm{l}$ of MTT ( $5 \mathrm{mg} / \mathrm{ml}$ ) solution was added to each well. After 4 hours of incubation, the remaining MTT solution was removed and $150 \mu \mathrm{l}$ of DMSO was added to each well, under standard assay conditions, shaking shaker on the low-speed shock for 5 minutes to completely dissolve the precipitation. After the formazan crystals had dissolved, the optical density (OD) was measured by enzyme-linked immunosorbent assay at $490 \mathrm{~nm}$. The cell inhibitory rate was calculated according to the following equation: the cell inhibitory rate $=[1-\mathrm{OD}$ experiment/OD control] $\times 100 \%$. All the experiments were performed three times.

\section{Statistical Analysis}

The experiments were performed in triplicate. Statistical analyses were carried out using the statistical software SPSS 20.0. Pearson's correlation coefficient (r) was calculated. The difference between the experimental groups and the control group was analyzed by one-way analysis of variance (ANOVA) followed by Dunnett's t-test. The statistically significant difference was considered to ${ }^{\star} \mathrm{P} \leq$ 0.05 compared to control cells and ${ }^{\star *} \mathrm{P} \leq 0.01$ compared to control cells.

\section{Result}

Figure 1 shows that the excitation wavelength of CdTe aqueous solution with concentration of $8 \mathrm{umol} / \mathrm{L}(3 \mathrm{mg} / \mathrm{ml}$ ) (calculated by UV absorption and Lambert Beer's law) is $450 \mathrm{~nm}$.

The emission wavelength is $537 \mathrm{~nm}$ (due to different detection conditions, the wavelength will fluctuate up and down $5 \mathrm{~nm}$ ).

Figure 2(a) and Figure 2(b) had the similar pattern. The inhibition rate of Cys-CdTe/ZnS QDs and Cys-CdTe QDs increased with the increase of concentration and reached maximum for $48 \mathrm{~h}$ (66.72\% for Figure 2(a), 57.01\% for 

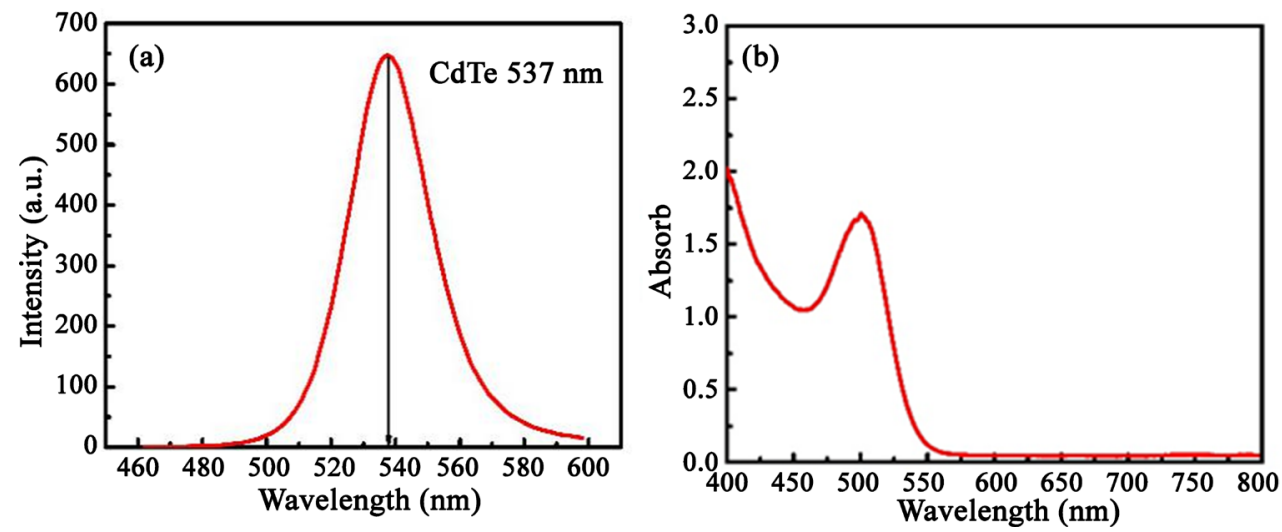

Figure 1. Fluorescence spectrum and UV absorption spectra of CdTe aqueous solution. (a) Fluorescence spectrum; (b) UV absorption spectrum.
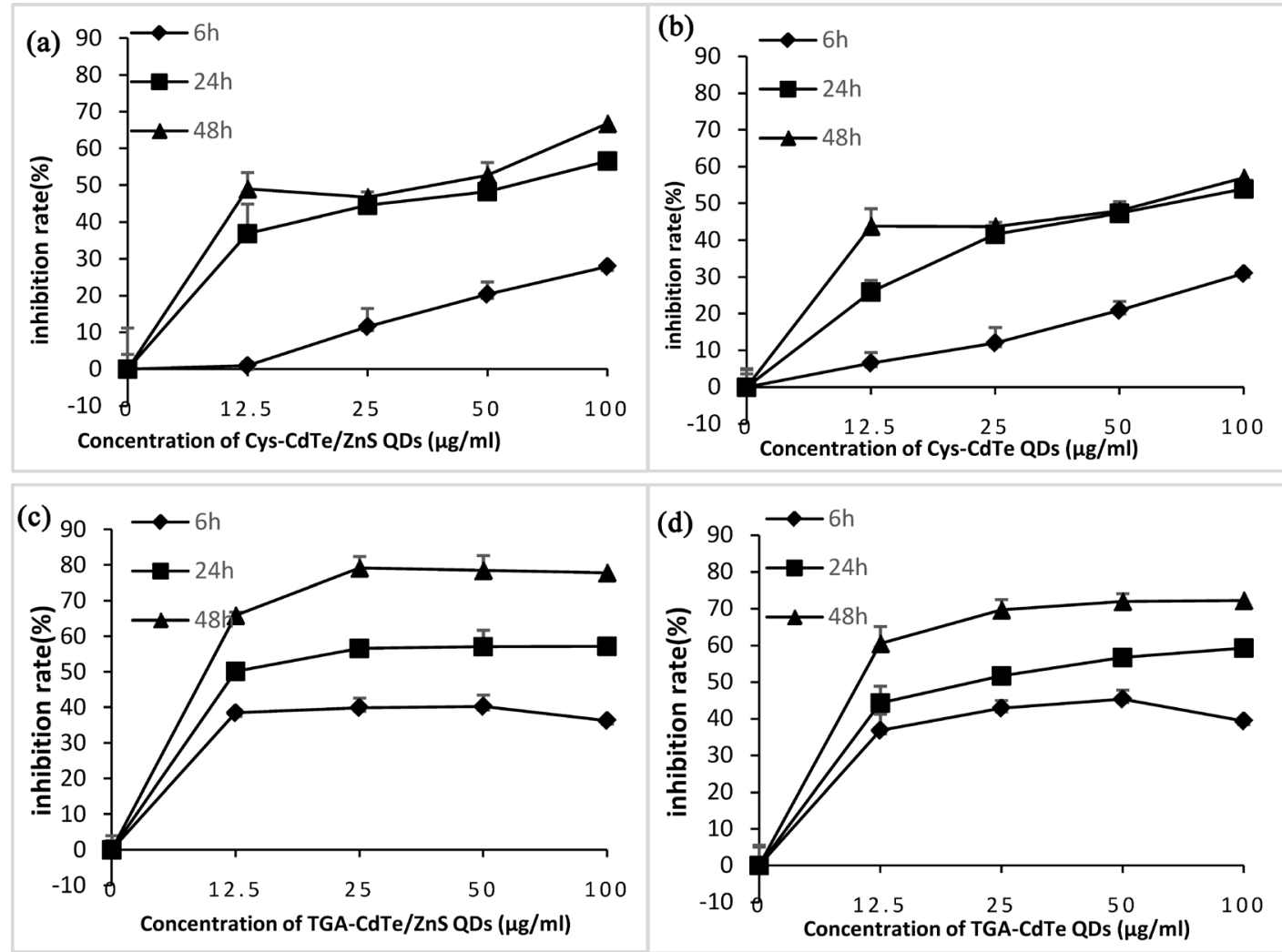

Figure 2. Caco-2 cells were treated to four CdTe-QDs for 6, 24 and 48 hours. The inhibition rate of Cys-CdTe/ZnS QDs (a); The inhibition rate of Cys-CdTe QDs (b); The inhibition rate of TGA-CdTe/ZnS QDs (c); The inhibition rate of TGA-CdTe QDs (d).

Figure $2(\mathrm{~b}))$. The inhibition rate $(0.96 \%-27.86 \%$ for Figure $2(\mathrm{a}), 6.54 \%-$ $30.92 \%$ for Figure 2(b)) was linear positive correlation at $6 \mathrm{~h}(\mathrm{r}=0.95, \mathrm{P} \leq 0.05$ for Figure 2(a), r = 0.98, P $\leq 0.05$ for Figure 2(b)). The inhibition rate increased with the increase of time and was linear positive correlation at the concentration of $12.5 \mu \mathrm{g} / \mathrm{ml}(\mathrm{r}=0.96 \mathrm{P} \leq 0.05$ for Figure 2(a), $\mathrm{r}=1.00 \mathrm{P} \leq 0.05$ for Figure 2(b)). The inhibition rate was almost similar with concentrations from 25 to 100 $\mu \mathrm{g} / \mathrm{ml}$ at $24 \mathrm{~h}$ and $48 \mathrm{~h}$. Figure 2(c) and Figure 2(d) had the similar pattern. 
The inhibition rate of TGA modified QDs reached maximum $65.88 \%$ at the lowest concentration with a slight fluctuate until $100 \mathrm{~g} / \mathrm{ml}$ at three exposure times. The inhibition rates both reached maximum for $48 \mathrm{~h}$ (79.21\% for Figure 2(c), $72.24 \%$ for Figure 2(d)).

Figure 3(a) showed the relative ratio of Cys-CdTe/ZnS QDs to Cys-CdTe QDs (Cys-ZnS/Cys). The ratios were closed to 1 (0.901 - 1.07) with concentrations from 25 to $100 \mu \mathrm{g} / \mathrm{ml}$ for $6 \mathrm{~h}$ and $24 \mathrm{~h}$. The average ratios of Cys-ZnS/Cys reached $1.11(1.07-1.17)$ for $48 \mathrm{~h}$. The lowest ratio reached 0.15 at concentration of $12.5 \mu \mathrm{g} / \mathrm{mll}$ for $6 \mathrm{~h}$. The largest ratio reached 1.4 at the concentration of $12.5 \mu \mathrm{g} / \mathrm{ml}$ for $24 \mathrm{~h}$. Figure 3 (b) showed the relative ratio of TGA-CdTe/ZnS QDs to TGA-CdTe QDs (TGA-ZnS/TGA). The ratio in all concentrations were closed to $1(0.89-1.14)$ at three exposure times.

Figure 4(a) showed the relative ratio of Cys-CdTe/ZnS QDs to TGA-CdTe QDs (Cys-ZnS/TGA-ZnS). Figure 4(b) showed the relative ratio of Cys-CdTe QDs to TGA-CdTe QDs (Cys/TGA). Figure 4(a) and Figure 4(b) had the similar pattern. The relative ratio is less than 1 in all concentrations at three exposure times. With the concentration increases, the ratio significant increased and exhibited linear positive correlation for $6 \mathrm{~h}(\mathrm{r}=0.97, \mathrm{P} \leq 0.01$ for Figure $4(\mathrm{a}), \mathrm{r}=0.99, \mathrm{P} \leq$

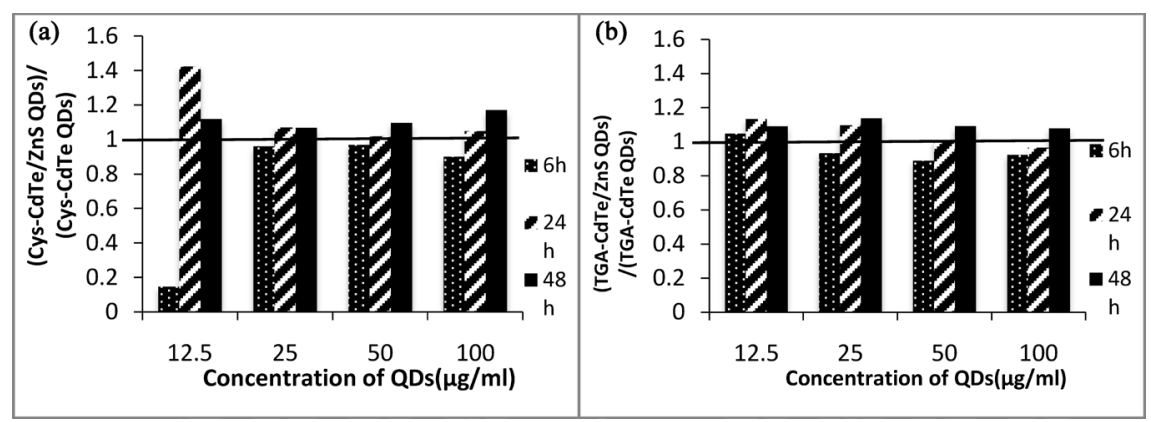

Figure 3. The relative ratio of CdTe-QDs with $\mathrm{ZnS}$ to $\mathrm{CdTe}-\mathrm{QDs}$ without $\mathrm{ZnS}$ on inhibition rate. The relative ratio of Cys-CdTe/ZnS QDs to Cys-CdTe QDs on inhibition rate (a); The relative ratio of TGA-CdTe/ZnS QDs to TGA-CdTe QDs on inhibition rate (b).
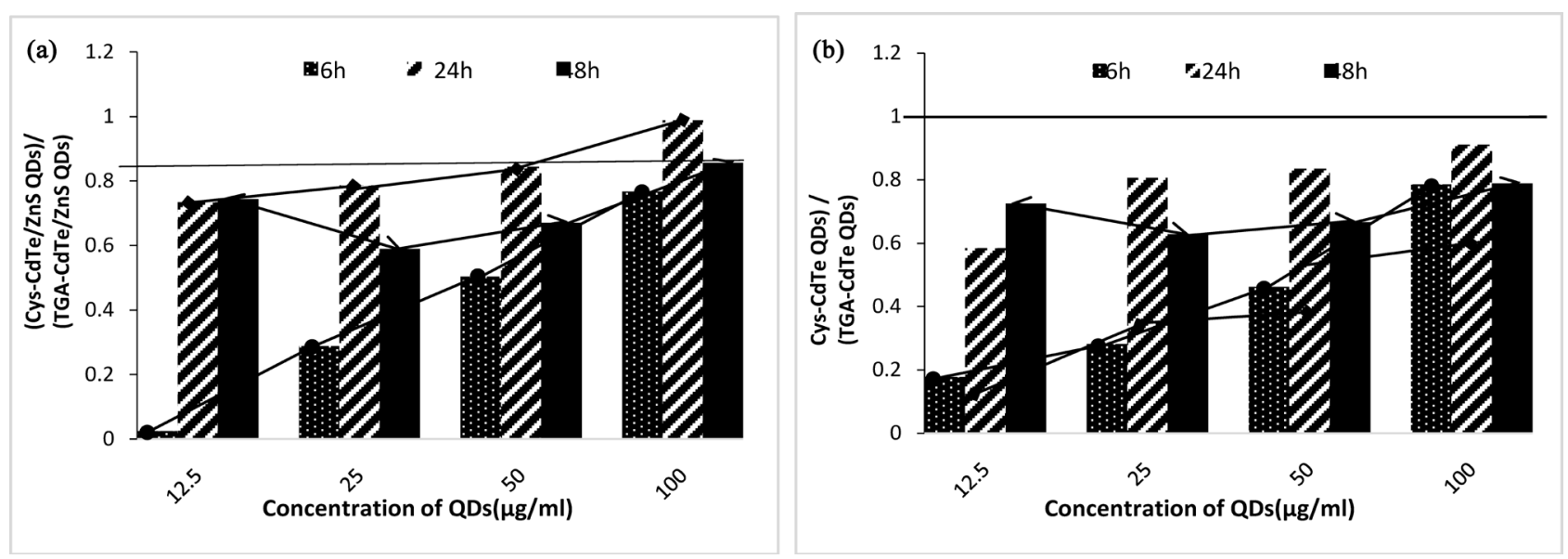

Figure 4. The comparison between Cys-modified QDs and TGA-modified QDs. The relative ratio of Cys-CdTe/ZnS QDs and TGA-CdTe/ZnS QDs inhibition rate (a); The relative ratio of Cys-CdTe QDs and TGA-CdTe QDs inhibition rate (b). 
0.01 for Figure 4(b)). With the concentration increases, the ratio increased slowly for $24 \mathrm{~h}$. The ratios reached lowest ( 0.025 for Figure 4(a), 0.18 for Figure 4 (b)) at concentration of $12.5 \mu \mathrm{g} / \mathrm{ml}$ for $6 \mathrm{~h}$. The ratios were closed to 0.85 at maximum concentration $(100 \mu \mathrm{g} / \mathrm{ml})$ at three exposure times. Figure 4 (b) exhibited time-dependent at concentration of $12.5 \mu \mathrm{g} / \mathrm{ml}(\mathrm{r}=0.951, \mathrm{P} \leq 0.05)$.

Table 1 showed the t-test result on the inhibition rate of Cys- $\mathrm{ZnS} / \mathrm{Cys}$, TGA-ZnS/TGA, Cys-ZnS/TGA-ZnS and Cys/TGA. The ratios of Cys-ZnS/Cys and TGA-ZnS/TGA had significant differences at high concentrations for $48 \mathrm{~h}$ $(\mathrm{P} \leq 0.05)$. The ratios of Cys-ZnS/TGA-ZnS and Cys/TGA had significant differences at most concentrations $(\mathrm{P} \leq 0.05)$ at three exposure times.

\section{Discussion}

Our result showed that four QDs (CdTe-QDs, TGA and Cys-modified CdTe/ZnS and CdTe QDs) have inhibited cell proliferation. A study reported CdTe QDs have inhibited to HepG2 and HELF cells proliferation and have dose dependent

Table 1. The t-test result on the inhibition rate of Cys-CdTe/ZnS QDs to Cys-CdTe QDs, TGA-CdTe/ZnS QDs to TGA-CdTe QDs, Cys-CdTe/ZnS QDs to TGA-CdTe/ZnS QDs and Cys-CdTe QDs to TGA-CdTe QDs.

\begin{tabular}{|c|c|c|c|c|c|c|c|c|}
\hline & \multicolumn{8}{|c|}{ t-test on the inhibition } \\
\hline & \multicolumn{2}{|c|}{ Cys-ZnS/Cys } & \multicolumn{2}{|c|}{ TGA-ZnS/TGA } & \multicolumn{2}{|c|}{ Cys-ZnS/TGA-ZnS } & \multicolumn{2}{|c|}{ Cys/TGA } \\
\hline & $\mathrm{t}$ & $\mathbf{P}$ & $\mathbf{t}$ & $\mathbf{P}$ & $\mathrm{t}$ & $\mathbf{P}$ & $\mathrm{t}$ & $\mathbf{P}$ \\
\hline \multicolumn{9}{|c|}{$6 \mathrm{~h}(\mu \mathrm{g} / \mathrm{ml})$} \\
\hline 0 & - & - & - & - & - & - & - & - \\
\hline 12.5 & -2.561 & 0.063 & 0.527 & 0.626 & -26.195 & $0.000^{* *}$ & -8.387 & $0.001^{* *}$ \\
\hline 25 & -0.277 & 0.795 & -1.106 & 0.331 & -37.874 & $0.000^{* *}$ & -10.162 & $0.001^{* *}$ \\
\hline 50 & -0.170 & 0.873 & -2.588 & 0.063 & -6.084 & $0.004^{* *}$ & -9.018 & $0.001^{* *}$ \\
\hline 100 & -1.257 & 0.277 & -1.313 & 0.259 & -3.122 & $0.035^{*}$ & -4.302 & $0.013^{*}$ \\
\hline \multicolumn{9}{|c|}{$24 \mathrm{~h}(\mu \mathrm{g} / \mathrm{ml})$} \\
\hline 0 & - & - & - & - & - & - & - & - \\
\hline 12.5 & 1.599 & 0.194 & 1.673 & 0.170 & -2.003 & 0.170 & -4.420 & $0.012^{*}$ \\
\hline 25 & 0.580 & 0.593 & 1.725 & 0.160 & -2.519 & 0.114 & -3.144 & $0.035^{\star}$ \\
\hline 50 & 0.855 & 0.441 & 0.516 & 0.633 & -17.555 & $0.000^{* *}$ & -7.655 & $0.002^{* *}$ \\
\hline 100 & 1.375 & 0.241 & -0.783 & 0.478 & -0.225 & 0.833 & -3.758 & 0.063 \\
\hline \multicolumn{9}{|c|}{$48 \mathrm{~h}(\mu \mathrm{g} / \mathrm{ml})$} \\
\hline 0 & - & - & - & - & - & - & - & - \\
\hline 12.5 & 1.390 & 0.237 & 1.471 & 0.215 & -5.142 & $0.007^{* *}$ & -4.064 & $0.015^{* *}$ \\
\hline 25 & 0.810 & 0.463 & 3.471 & $0.026^{*}$ & -12.414 & $0.000^{* *}$ & -6.806 & $0.002^{* *}$ \\
\hline 50 & 4.302 & $0.013^{*}$ & 2.710 & 0.054 & -12.842 & $0.000^{* *}$ & -13.906 & $0.000^{* *}$ \\
\hline 100 & 3.850 & $0.018^{\star}$ & 2.078 & 0.106 & -3.500 & $0.025^{\star}$ & -8.061 & $0.001^{* *}$ \\
\hline
\end{tabular}

${ }^{*} \mathrm{P} \leq 0.05 ;{ }^{* *} \mathrm{P} \leq 0.01$. 
and time dependent [10], which supports our observations.

It has reported that cytotoxicity of QDs depend on their surface modification [11]. Our result showed that the inhibition rate of TGA-modified QDs was higher than Cys-modified QDs. For TGA-modified QDs, the inhibition on cell proliferation exhibited obviously time-dependent and no effected by design-concentration range. The inhibition was almost consistent in all concentrations and had already reached this level at the lowest concentration. At a low concentration, TGA-CdTe QDs also showed the cytotoxic effects to HepG2 cells [12]. Contrary to TGA, Cys-modified QDs of the inhibition exhibited obviously dose-dependent and no obviously time-dependent. The inhibition was similar at $24 \mathrm{~h}$ and $48 \mathrm{~h}$. The inhibition was almost no effect at the designed-lowest concentration and shortest time. At a low concentration and short time, Cys-CdTe QDs also had no apparent effect in cell metabolic activity and apoptosis [13].

The chemical composition of the QDs core is important factors affecting QDs toxicity [14]. For Cys-modified QDs, the inhibition of CdTe/ZnS QDs was higher than CdTe QDs at the higher concentration in the maximum time despite they both inhibited to cell proliferation. For TGA-modified QDs, the inhibition of CdTe/ZnS QDs and CdTe QDs were almost consistent at all concentrations and three exposure times.

\section{Conclusion}

We found that four CdTe-QDs, modified with TGA or Cys, with ZnS core or not, all have inhibited cell proliferation. The inhibition of TGA-modified QDs was higher than Cys-modified QDs. Inhibition exhibited obviously time-dependent for TGA-modified QDs while exhibited obviously dose-dependent for Cys-modified QDs. The inhibition of CdTe/ZnS QDs was higher than CdTe QDs for Cys-modified QDs. The inhibition of CdTe/ZnS QDs was almost consistent at all concentrations and three exposure times. The study facilitates the further understanding of the inhibition effect on cell proliferation of CdTe QDs with core or functional group and provides useful information for the use of CdTe QDs.

\section{Funding}

This research was financially supported by the National Natural Science Foundation of China (Grant No. 31460249) and the science plan project of Inner Mongolia Autonomous Region (Grant No. 201505016).

\section{Conflicts of Interest}

The authors declare no conflicts of interest regarding the publication of this paper.

\section{References}

[1] Nguyen, K.C., Rippstein, P., Tayabali, A.F., et al. (2015) Mitochondrial Toxicity of Cadmium Telluride Quantum Dot Nanoparticles in Mammalian Hepatocytes. Tox- 
icological Sciences, 146, 31-42. https://doi.org/10.1093/toxsci/kfv068

[2] Zhang, Y.J. and Clapp, A. (2011) Overview of Stabilizing Ligands for Biocompatible Quantum Dot Nanocrystals. Sensors, 11, 11036-11055.

https://doi.org/10.3390/s111211036

[3] Rizvi, S.B., Yildirimer, L., Ghaderi, S., et al. (2012) A Novel POSS-Coated Quantum Dot for Biological Application. International Journal of Nanomedicine, 7, 3915-3927. https://doi.org/10.2147/IJN.S28577

[4] Hardman, R. (2006) A Toxicologic Review of Quantum Dots: Toxicity Depends on Physicochemical and Environmental Factors. Environmental Health Perspectives, 114, 165-172. https://doi.org/10.1289/ehp.8284

[5] Nguyen, K.C., Seligy, V.L. and Tayabali, A.F. (2013) Cadmium Telluride Quantum Dot Nanoparticle Cytotoxicity and Effects on Model Immune Responses to Pseudomonas aeruginosa. Nanotoxicology, 7, 202-211. https://doi.org/10.3109/17435390.2011.648667

[6] Zheng, H., Mortensen, L.J. and Delouise, L.A. (2013) Thiol Antioxidant-Functionalized CdSe/ZnS Quantum Dots: Synthesis, Characterization, Cytotoxicity. Journal of Biomedical Nanotechnology, 9, 382-392. https://doi.org/10.1166/jbn.2013.1561

[7] Liu, N., Mu, Y., Chen, Y., et al. (2013) Degradation of Aqueous Synthesized CdTe/ZnS Quantum Dots in Mice: Differential Blood Kinetics and Biodistribution of Cadmium and Tellurium. Particle and Fibre Toxicology, 10, Article No. 37.

https://doi.org/10.1186/1743-8977-10-37

[8] Du, T., Cai, K.M., Han, H.Y., et al. (2015) Probing the Interactions of CdTe Quantum Dots with Pseudorabies Virus. Scientific Reports, 5, Article No. 16403. https://doi.org/10.1038/srep16403

[9] Zhang, T., Hu, Y.Y., Meng, T., et al. (2015) Liver Toxicity of Cadmium Telluride Quantum Dots (CdTe QDs) Due to Oxidative Stress in Vitro and in Vivo. International Journal of Molecular Sciences, 16, 23279-13299. https://doi.org/10.3390/ijms161023279

[10] Xu, P., Li, J., Shi, L., et al. (2013) Synergetic Effect of Functional Cadmium-Tellurium Quantum Dots Conjugated with Gambogic Acid for HepG2 Cell-Labeling and Proliferation Inhibition. International Journal of Nanomedicine, 8, 3729-3736. https://doi.org/10.2147/IJN.S51622

[11] Hoshino, A., Fujioka, K., Oku, T., et al. (2004) Physicochemical Properties and Cellular Toxicity of Nanocrystal Quantum Dots Depend on Their Surface Modification. Nano Letters, 4, 2163-2169. https://doi.org/10.1021/nl048715d

[12] Wang, L., Zhang, H., Lu, C., et al. (2014) Ligand Exchange on the Surface of Cadmium Telluride Quantum Dots with Fluorosurfactant-Capped Gold Nanoparticles: Synthesis, Characterization and Toxicity Evaluation. Journal of Colloid and Interface Science, 413,140-146. https://doi.org/10.1016/j.jcis.2013.09.034

[13] Wu, C., Shi, L., Li, Q., et al. (2010) Probing the Dynamic Effect of Cys-CdTe Quantum Dots toward Cancer Cells In Vitro. Chemical Research in Toxicology, 23, 82-88. https://doi.org/10.1021/tx900291c

[14] Cho, S.J., Maysinger, D., Jain, M., et al. (2007) Long-Term Exposure to CdTe Quantum Dots Causes Functional Impairments in Live Cells. Langmuir, 23, 1974-1980. https://doi.org/10.1021/la060093j 